\title{
Transcranial direct current stimulatio (tDCS) and transcranial current alternating stimulation (tACS) review
}

\author{
M. Rodríguez-Ugarte, Nadia Sciacca, José M. Azorín \\ Brain-Machine Interface Systems Lab, Miguel Hernández University \\ Av. de la Universidad S/N, 03202 Elche \\ maria.rodriguezu@umh.es, nadiascih@gmail.com,jm.azorin@umh.es
}

\begin{abstract}
This literature review is aimed to explore the main technical characteristics of both transcranial direct current stimulation (tDCS) and transcranial alternate current stimulation (tACS) using the latest research on both healthy and impaired subjects. These techniques have no official standards developed yet. Our intent is to underline the main properties and problems linked with the application of those techniques which show diverse, and sometimes even opposite, results depending mainly on electrode positioning and underlying brain activity.
\end{abstract}

Key words: transcranial direct current stimulation (tDCS), transcranial alternate current stimulation (tACS), long term potentiation (LTP), excitatory post synaptic potential (EPSP), primary motor cortex (M1), motor evoked potential (MEP), stroke.

\section{Introduction}

Among different impairments that can affect standard brain functions, we choose to focus primarily on stroke, because it is one of the most prevalent and severe disability worldwide [1]. It is known that after a cerebrovascular accident, reorganization of neural tissues takes place [18]. If the ischemic event occurs on the motor area and it is severe enough to block the spontaneous neural reorganization, it could lead to paresis or even paralysis of one or more body parts [24].

In order to ameliorate stroke rehabilitation, different approaches have been carried out. Over the last decade, within the field of functional rehabilitation, transcranial current stimulation (tCS) has garnered considerable attention. It is assumed to improve, above other, motor functions in both healthy and stroke individuals [25], [4], [23].

There are three different types of tCS: transcranial direct current stimulation (tDCS), transcranial alternate current stimulation (tACS) and random noise stimulation (tRNS). All of them are noninvasive and involve low intensity current induction into the brain. Some studies have investigated the physiological basis of tDCS and tACS in order to get the picture of standard pattern that can be used for future research [36], [32].

This paper is oriented towards a broad audience who wants to understand the basic mechanisms of tDCS and tACS techniques. The main parameters of each type of stimulation and the implications related to its application on healthy subjects, stroke patients and individuals with unusual brain oscillations are discussed.

\section{TDCS and TACS Properties}

\section{$2.1 \quad$ tDCS}

The main characteristic of tDCS resides on the capacity to modulate neurons membrane resting potential. It has been demonstrated that tDCS alters cortical excitability of the brain [26]. The scientific community, intends to discover how to modulate this for long-lasting effects and therefore, to strengthen the synaptic connection [16], [36]. Long term potentiation (LTP) could occur if spaced stimulation sessions are performed repeatedly [42]. In other words, if the membrane resting potential is depolarized, it would be easier for the neurons to fire [36].

Simply put, it is expected that the electrical induction provokes voltage-gated sodium channels along the axon to open, leading voltage-gated potassium channels to release potassium ions along the axon. The signal travels from one neuron to the next one thanks to voltage-dependent calcium channels, which regulate among other things, the release of the neurotransmitters from one cell to another. This will cause the synaptic vesicles to fused with the plasma membrane in order to release the neurotransmitters into the synaptic cleft. If for instance, the neurotransmitter is glutamate and the stimulus is given with sufficient strength, the glutamate will go inside the postsynaptic neuron depolarizing it and causing an excitatory postsynaptic potential (EPSP). When this process is repeated many times, it will produce LTP.

Therefore, if the neurons' threshold is more positive it would be easier to induce action potentials 
hence, to increase the firing rate. This would fulfill Hebb's theory about LTP: "When an axon of cell $\mathrm{A}$ is near enough to excite cell $\mathrm{B}$ and repeatedly or persistently takes part in firing it, some growth process or metabolic change takes place in one or both cells such that A's efficiency as one of the cells firing B is increased" [11].

However, when it comes to apply tDCS, the effects of the stimulation are determined by different parameters: area of the electrode, current intensity applied, duration of the stimulation and polarization of the electrodes. All of them play an important role in the desirable results. For instance, it is not the same to perform a cathodal stimulation which decreases cortical excitability than an anodal one that increases excitability [36],[6]. Nevertheless, Purpura and McMurtry [34] found that anodal stimulation deactivated neurons in deeper layers and cathodal activate them. On the other hand, there are contrasting studies that performed anodal stimulation on the leg motor area (which include deep layers of the brain) obtaining excitatory effects in the lumbar spinal network or improvements of the foot pinch force [38], [41].

Moreover, both, the area of the electrodes and the intensity applied, define the current density, which is $\operatorname{Amperes}(\mathrm{A}) / \operatorname{Area}(\mathrm{S})$. This parameter should be inside a safety limit to avoid neural or tissue damage [29]. Also, the duration of the stimulation itself is an important value for the after effects which could vary from few seconds to almost one hour [36].

\subsection{Transcranial Alternate Current Stimulation}

Cortical rhythms of the brain are produced by the electrical activity from the neurons and, as it was explained before, they can generate EPSP. The sum of EPSP are the brain waves recorded by EEG from the scalp [34], [28]. Brain waves can be classified in 4 main bands: delta band $(<4$ $\mathrm{Hz})$, theta band $(4-7 \mathrm{~Hz})$, alpha band $(8-15$ $\mathrm{Hz}$ )and beta band $(16-31 \mathrm{~Hz})[7]$.

tACS is a non invasive brain stimulation technique which applies weak sinusoidal currents from the scalp. The basic principal of tACS is to interfere with these cortical rhythms and modulate the cerebral cortex excitability in a frequency-specific manner [2], [45], [12]. There are three main parameters that characterize tACS: the frequency, the intensity and the phase.

Focussed on a study by Garside [10], $4 \mathrm{~Hz}$ tACS corroborated the hypothesis that the disruption of low frequencies oscilations generation is related to interruption in declarative memory consolidation. In addition, tACS at this frequency was applied in a study performed by Riecke [35], finding that this technique can affect auditory perception. Moreover, in other study, dizziness in the parietal area was stronger at $4 \mathrm{~Hz}$ than at other frequencies [37].

In a study by Moliadze [27], different types of intensity were applied. Finding no effects with 0.2 $\mathrm{mA}$; inhibition with $0.4 \mathrm{~mA}$ and no significant effects 0.6 and $0.8 \mathrm{~mA}$. However, in another study by Kanai [19], they applied $0.75 \mathrm{~mA}$ tACS observing a decrease in the phosphene threshold and concluding that tACS can selectively modulate the excitability of the occipital cortex.

Furthermore, how the phase of a wave could affect the coherence of the beneath oscillatory neural activity is explained in a study by Thut [43]. When both signals are synchronized, there is an increased in coherence, whereas the opposite effect can be obtained when the waves are induced out of synchrony.

\section{$3 \quad$ TDCS and TACS Applications}

\section{1 tDCS in healthy population}

It was demonstrated that anodal-tDCS applied over one side of the primary motor cortex, along the homunculus motorious, facilitates motor function of contralateral part of the body [41], [6]. Similarly, applying cathodal-tDCS a reverse effect was observed [22]. However, a fundamental question remain regarding the optimal electrode configuration on the scalp. In order to answer this question many studies applied many different montages.

A study by Kidgell and colleagues [20] involved anodal-tDCS over the right M1 applying unilateral stimulation, whilst in a second condition they used cathodal-tDCS over the left M1 and anodaltDCS over the right M1. Bilateral stimulation was applied in order to find out if unilateral or bilateral stimulation would diversely modulate the motor functions of the non-dominant hand. By eliciting motor evoked potentials(MEPs)they have reported no difference between unilateral and bilateral stimulation with a significant increment in corticomotor excitability in both conditions $(\mathrm{P}<$ $0.05)$.

Another question that is often asked regarding the efficacy of the tDCS is related with the level of engagement of the subject during the stimulation process. To investigate this variable, Antal and collegues [3] created three experimental conditions. In the first one, the subjects were required to sit passively during the stimulation, in the sec- 
ond one, the subject's attention was directed towards a cognitive task and in the last one, the subjects were instructed to push a ball with their right hand. MEPs were recorded before and after 10 min of anodal and cathodal stimulation. Their results shown that plastic changes in M1 were detectable on both conditions; if subjects were mentally engaged and when performing repeated contractions of a target muscle but not in the passive condition. Hence, tDCS efficacy relays also on the state of the subject during stimulation.

Most of the papers that investigate tDCS effects over the motor cortex applying anodal current, correlate the enhanced cortical excitability to improved motor performance [30]. This suggests that excitability itself can be involved in the plastic-learning process. Nevertheless, we have to take into account that each subject could display different pattern of activations and that if we move from healthy subjects to impaired individuals we may not be able to apply the same theoretical inferences about tDCS applications and outcomes.

\section{2 tDCS in stroke population}

When it comes to apply tDCS on patients which suffered a brain lesion, no universal methodologies subsist. Furthermore, lesioned brain tissue can influence the current flow, thus is highly probable that an experimental paradigm can work for some patients but cannot for others. In a study by Lefebvre et. al. [23] experimented on eighteen chronic stroke patients. They observed that ten patients rapidly and significantly enhanced online motor skill learning, successfully translating the improvement into long-term retention and generalization of the motor skill. However, the other eight patients showed online deterioration of performance. Among those eight, four steadily worsened from the beginning and the other half started to improve but worsened later. The anode was positioned over the ipsilesional M1, while the cathode over the contralesional speculative region. Dual tDCS was applied while learning a complex motor skill with the paretic hand. The observed deterioration could be due to diverse factors as: fatigue, poor attention, inefficiency in motor skill learning network engagement, or even the stimulation itself could be the cause of a greater imbalance of interhemispheric excitability.

It has to be considered that dual or cathodal tDCS on the contralesional motor cortex may be deleterious in the most severely impaired stroke patients [23]. In another study on chronic stroke subjects by Hummel and Cohen [15] instead, we can note that even if tDCS did not affect significantly motor thresholds (MT) when compared with healthy subjects, resulted anyway reported a functional improvement of the paretic hand in all patients. The anode in this case was placed over ipsilesional M1 hand area, while the cathode was placed on the contralateral supraorbital region.

The severity of subjects's impairment can deeply influence the degrees of rehabilitation regained. Hesse and collegues [13] from their side, conducted an experiment on 96 patients with post-stoke severe upper limb paresis, finding very different results. They experimented for six weeks in three groups of patients. One group received sham stimulation, the second and the third group received stimulation for 20 minutes at $2 \mathrm{~mA}$, diverging just in current polarity (anodal versus cathodal).

The stimulation electrode was placed over the hand area and the return electrode was placed above the contralateral orbit. They observed no effects neither with anodal nor with cathodal stimulation for arm training in this population of stroke patients with cortical involvement and severe weakness. However, we have to underline that no standard protocols for electrode montage, current intensity, time and pattern of stimulation has been established yet for impaired subjects as for healthy ones. Many variables may have played an influential role in the experiments previously mentioned to produce such different results.

Moreover, the stroke population itself is a very heterogeneous sample hence, more experiments need to be performed to get unequivocal answers about the optimal protocols to apply.

\section{3 tACS on healthy subjects}

The rational to apply tACS stimulation relays on the fact that neurons naturally engage in different oscillatory activities in relation with each specific cognitive tasks. A prevalent oscillatory activity of the motor system occurs in the $B$ frequency band $(13-30 \mathrm{~Hz})$ [33]. In Healthy subjects, it is possible to observe $\beta$ band increasing while sustaining tonic contractions and being suppressed during and before starting voluntary movements. Pogosyan et. al. [33] applied tACS on fourteen healthy subjects performing a visuomotor task. The current was delivered at $20 \mathrm{~Hz}$, with an intensity ranging from 0.1 to $0.2 \mathrm{~mA}$, over the hand M1 area while the return electrode was positioned over the ipsilateral side of the neck. Their results showed that 20 $\mathrm{Hz}$ tACS slow voluntary movements. Meanwhile, applying $5 \mathrm{~Hz}$ tACS they found no results.

Another study in agreement with the observation of movement slowing after $20 \mathrm{~Hz}$ tACS administration comes from Wach et. al. [44]. The researchers applied both 10 and $20 \mathrm{~Hz}$ tACS on 
15 healthy subjects for 10 minutes, in order to investigate movement speed and accuracy of the right hand. They detected an increase in movement variability after $10 \mathrm{~Hz}$ tACS administration, especially in task requiring internal pacing, while $20 \mathrm{~Hz}$, in agreement with other studies, induced movement slowing [17]. An interesting exploratory study about 1, 5, 10, 15, 20, 30 and $45 \mathrm{~Hz}$ tACS effects over M1, for a stimulation period from 2 to 5 minutes, was performed by Schutter and Hortensius [39] on eight healthy volunteers. They stimulated for one session the hot spot over M1, found where the lowest intensity was required for $50 \%$ thumb movements by using TMS pulses. They identified significant increases in motor evoked potentials(MEP) amplitude after 5 and $20 \mathrm{~Hz}$ tACS over both left and right M1 compared to baseline, but did not observe any significant changes in the other frequency bands.

An additional confirmation about $20 \mathrm{~Hz}$ tACS effects comes from Joundi et. al.[17] with a further interesting observation about $70 \mathrm{~Hz}$ tACS effects on M1. Their goal was to test, on healthy subjects, the anti-kinetic beta band and the prokinetic gamma band. They detected an increase in frontal gamma activity linked with a reduction in reaction times. But it is possible for those correspondences to reflect an epiphenomena rather than a causal relation.

\section{4 tACS in medical population}

tACS application on particular medical population have been found to be a promising tool for rehabilitation purposes. In an experiment by Brittain et. al. [5] for example, they investigated whether using the basic tremor rhythm of each patients or it's first harmonic according to the stimulation paradigm, could lead to reduce the intensity of the tremor. They used peripheral tremor amplitude as a proxy for cortical oscillatory activity in order to identify phase dependency to induce cortical phase cancellation in twelve Parkinson's patients. Their results revealed significant attenuation of Parkinsonian rest tremor, achieving almost $50 \%$ average reduction in amplitude, using both the basic and the first harmonic rhythm. Even if the ideal suppressive phase persisted for 30 sec it still remains a promising first step towards a deeper understanding of brain waves modulation and more importantly an alternative tool for Parkinson's patients who are non eligible for deep brain stimulation. Moreover the phase cancellation approach could be a valid tool for other kind of oscillopathies.

Nevertheless, transcranial current stimulation opens the possibility for an alternative applica- tion for drugs resistant therapies population. One of the latest example comes from a study of Kallel and colleagues [21], where they applied $4.5 \mathrm{~Hz}$ tACS bifrotally on three schizophrenic patients for 20 sessions. They reported a boost in patient's insight about the illness, a decline in negative symptoms and a generalized decrease also in positive symptoms. The rational for this choice relies on antecedent studies where tDCS was applied over the left dorsolateral prefrontal cortex alleviating negative schizophrenic symptoms [31].

\section{Conclusion}

There is evidence that tDCS and tACS are capable of modulating the brain activity and moreover, of inducing physical and memory enhancement in certain population of subjects.

Nevertheless, there are no standard protocols developed by the scientific community yet. Hence, the electrode configuration, the intensity and the frequency adopted to reach the desirable results are quite variable. This is understandable in the context that the research is in its early phase, where each experimental result represents a basis for the following investigations to accept or discard certain parameters.

While tDCS modulates neurons membrane resting potential, tACS interferes with the brain waves to obtain the desired outcome. It has to be considered that tDCS has been investigated further than tACS. Thus, its parameters are better known and easier to apply than tACS ones.

When anodal tDCS is applied, it produces a greater excitatory response of the underlaying neurons, decreasing the excitatory threshold, while cathodal tDCS induces lower neural firing rates, increasing the excitatory threshold. For instance, a study performed by Tanaka [41] showed that it is possible to improve the performance of the pinch force of the lower limb in a healthy subject using tDCS induction. When it comes to stroke patients, the degree of impairment and the lesion sites vary widely. Indeed, the results of applying tDCS are as heterogeneous as its population.

However, there is a promising general trend in this population and further research could clear the boundaries between one group of patients and another in order to apply different rehabilitative strategies [13].

On the other hand, tACS research is still in its early phases and its applications need to be further investigated. Varying results have been observed when using $20 \mathrm{~Hz}$ tACS at different phases of a 
particular motor task [9], [44].

\section{Acknowledgement}

This research has been carried out in the framework of the project Associate - Decoding and stimulation of motor and sensory brain activity to support long term potentiation through Hebbian and paired associative stimulation during rehabilitation of gait (DPI2014-58431-C4-2-R), funded by the Spanish Ministry of Economy and Competitiveness and by the European Union through the European Regional Development Fund (ERDF) A way to build Europe.

\section{References}

[1] Adamson, J., Beswick, A. and Ebrahim, S., (2004) Is stroke the most common cause of disability?. Journal of Stroke and Cerebrovascular Diseases, 13(4), pp.171-177.

[2] Antal, A. and Paulus, W., (2013) Transcranial alternating current stimulation (tACS).

[3] Antal, A., Terney, D., Poreisz, C. and Paulus, W., (2007) Towards unravelling task-related modulations of neuroplastic changes induced in the human motor cortex. European Journal of Neuroscience, 26(9), pp 2687-2691.

[4] Berryhill, M.E. and Jones, K.T., (2012) tDCS selectively improves working memory in older adults with more education. Neuroscience letters, 521(2), pp.148-151.

[5] Brittain, J. S., Probert-Smith, P., Aziz, T. Z. and Brown, P., (2013) Tremor suppression by rhythmic transcranial current stimulation. Current Biology, 23(5), pp 436-440.

[6] Cogiamanian, F., Marceglia, S., Ardolino, G., Barbieri, S. and Priori, A., (2007) Improved isometric force endurance after transcranial direct current stimulation over the human motor cortical areas. European Journal of Neuroscience, 26(1), pp.242-249.

[7] da Silva, F.L., (1991) Neural mechanisms underlying brain waves: from neural membranes to networks. Electroencephalography and clinical neurophysiology, 79(2), pp 81-93.

[8] Fehér, K.D. and Morishima, Y., (2016) Concurrent Electroencephalography Recording During Transcranial Alternating Current Stimulation (tACS). Journal of visualized experiments: JoVE, (107).

[9] Feurra, M., Paulus, W., Walsh, V. and Kanai, R., (2011) Frequency specific modulation of human somatosensory cortex. Front Psychol, 2, p 13 .
[10] Garside, P., Arizpe, J., Lau, C.I., Goh, C. and Walsh, V., (2015) Cross-hemispheric alternating current stimulation during a Nap disrupts slow wave activity and associated memory consolidation. Brain stimulation, 8(3), pp.520-527.

[11] Hebb, D., (1968). 0. The organization of behavior.

[12] Helfrich, R.F., Schneider, T.R., Rach, S., Trautmann-Lengsfeld, S.A., Engel, A.K. and Herrmann, C.S., (2014) Entrainment of brain oscillations by transcranial alternating current stimulation. Current Biology, 24(3), pp 333-339.

[13] Hesse, S., Waldner, A., Mehrholz, J., Tomelleri, C., Pohl, M. and Werner, C., (2011) Combined transcranial direct current stimulation and robot-assisted arm training in subacute stroke patients an exploratory, randomized multicenter trial. Neurorehabilitation and neural repair, 25(9), pp 838-846.

[14] Hummel, F., Celnik, P., Giraux, P., Floel, A., Wu, W.H., Gerloff, C. and Cohen, L.G., (2005) Effects of non-invasive cortical stimulation on skilled motor function in chronic stroke. Brain, 128(3), pp 490-499.

[15] Hummel, F. C. and Cohen, L.G., (2006) Noninvasive brain stimulation: a new strategy to improve neurorehabilitation after stroke. The Lancet Neurology, 5(8), pp 708-712.

[16] Jeffery, D.T., Norton, J.A., Roy, F.D., and Gorassini, M.A., (2007) Effects of transcranial direct current stimulation on the excitability of the leg motor cortex. Exp Brain Res., 182, pp 281-287

[17] Joundi, R.A., Jenkinson, N., Brittain, J.S., Aziz, T.Z. and Brown, P., (2012) Driving oscillatory activity in the human cortex enhances motor performance. Current Biology, 22(5), pp 403-407.

[18] Kaas, J.H., Florence, S.L. and Jain, N., (1997) REVIEW: Reorganization of Sensory Systems of Primates after Injury. The Neuroscientist, 3(2), pp 123-130.

[19] Kanai, R., Paulus, W. and Walsh, V., (2010) Transcranial alternating current stimulation (tACS) modulates cortical excitability as assessed by TMS-induced phosphene thresholds. Clinical Neurophysiology, 121(9), pp 1551-1554.

[20] Kidgell, D.J., Goodwill, A.M., Frazer, A.K. and Daly, R.M., (2013) Induction of cortical 
plasticity and improved motor performance following unilateral and bilateral transcranial direct current stimulation of the primary motor cortex. BMC neuroscience, 14(1),pp 1.

[21] Kallel, L., Mondino, M. and Brunelin, J., (2016) Effects of theta-rhythm transcranial alternating current stimulation $(4.5 \mathrm{~Hz}-$ tACS) in patients with clozapine-resistant negative symptoms of schizophrenia: a case series. Journal of Neural Transmission, pp 15 .

[22] Lang, N., Nitsche, M.A., Paulus, W., Rothwell, J.C. and Lemon, R.N., (2004) Effects of transcranial direct current stimulation over the human motor cortex on corticospinal and transcallosal excitability. Experimental Brain Research, 156(4), 439-443,pp 1.

[23] Lefebvre, S., Laloux, P., Peeters, A., Desfontaines, P., Jamart, J. and Vandermeeren, Y., (2013) Dual-tDCS enhances online motor skill learning and long-term retention in chronic stroke patients. Frontiers in human neuroscience, 6, pp 343.

[24] Liepert, J., Bauder, H., Miltner, W.H., Taub, E. and Weiller, C., (2000) Treatment-induced cortical reorganization after stroke in humans. Stroke, 31(6), pp.1210-1216.

[25] Madhavan, S., Weber II, K.A. and Stinear, J.W., (2011) Non-invasive brain stimulation enhances fine motor control of the hemiparetic ankle: implications for rehabilitation. Experimental brain research, 209(1), pp 9-17.

[26] Minarik, T., Sauseng, P., Dunne, L., Berger, B. and Sterr, A., (2015) Effects of Anodal Transcranial Direct Current Stimulation on Visually Guided Learning of Grip Force Control. Biology, 4(1), pp 173-186.

[27] Moliadze, V., Atalay, D., Antal, A., and Paulus, W., (2012) Close to threshold transcranial electrical stimulation preferentially activates inhibitory networks before switching to excitation with higher intensities. BrainStimul. 5, pp 505-511.

[28] Mori, T. and Kai, S., (2002) Noise-induced entrainment and stochastic resonance in human brain waves. Physical Review Letters, 88(21), pp 218101.

[29] Nitsche, M.A., Liebetanz, D., Lang, N., Antal, A., Tergau, F. and Paulus, W., (2003) Safety criteria for transcranial direct current stimulation (tDCS) in humans. Clinical Neurophysiology, 114(11), pp.2220-2222.
[30] Nitsche, M. A., Schauenburg, A., Lang, N., Liebetanz, D., Exner, C., Paulus, W., Tergau, F., (2003) Facilitation of implicit motor learning by weak transcranial direct current stimulation of the primary motor cortex in the human. Journal of cognitive neuroscience, 15(4), pp 619-626.

[31] Palm, U., Keeser, D., Blautzik, J., Pogarell, O., Ertl-Wagner, B., Kupka, M. J. and Padberg, F., (2013) Prefrontal transcranial direct current stimulation (tDCS) changes negative symptoms and functional connectivity MRI (fcMRI) in a single case of treatmentresistant schizophrenia. Schizophrenia research, 150(2), pp 583-585.

[32] Pevzner, A., Izadi, A., Lee, D.J., Shahlaie, K. and Gurkoff, G.G., 2016. Making waves in the brain: What are oscillations, and why modulating them makes sense for brain injury. Frontiers in systems neuroscience, 10.

[33] Pogosyan, A., Gaynor, L. D., Eusebio, A. and Brown, P., (2009) Boosting cortical activity at beta-band frequencies slows movement in humans. Current Biology, 19(19), pp 16371641.

[34] Purpura, D.P. and McMurtry, J.G., (1965) Intracellular activities and evoked potential changes during polarization of motor cortex. Journal of Neurophysiology, 28(1), pp.166185.

[35] Riecke, L., Formisano, E., Herrmann, C.S. and Sack, A.T., (2015) 4-Hz transcranial alternating current stimulation phase modulates hearing. Brain stimulation, 8(4), pp 777783.

[36] Stagg, C.J. and Nitsche, M.A., (2011) Physiological basis of transcranial direct current stimulation. The Neuroscientist, 17(1), pp 3753 .

[37] Raco, V., Bauer, R., Olenik, M., Brkic, D. and Gharabaghi, A., (2014) Neurosensory effects of transcranial alternating current stimulation. Brain stimulation, 7(6), pp 823-831.

[38] Roche, N., Lackmy, A., Achache, V., Bussel, B. and Katz, R., (2011) Effects of anodal transcranial direct current stimulation over the leg motor area on lumbar spinal network excitability in healthy subjects. The Journal of physiology, 589(11), pp 2813-2826.

[39] Schutter, D. J. and Hortensius, R., (2011) Brain oscillations and frequency-dependent modulation of cortical excitability. Brain stimulation, pp 4(2), 97-103. 
[40] Steriade, M., Nuñez, A. and Amzica, F., (1993) Intracellular analysis of relations bet ween the slow (i $1 \mathrm{~Hz}$ ) neocortical oscillation and other sleep rhythms of the electroencephalogram. The Journal of Neuroscience, 13(8), pp 3266-3283.

[41] Tanaka, S., Hanakawa, T., Honda, M. and Watanabe, K., (2009) Enhancement of pinch force in the lower leg by anodal transcranial direct current stimulation. Experimental brain research, 196(3), pp 459-465.

[42] Trepel, C. and Racine, R.J. (2000) GABAergic modulation of neocortical long-term potentiation in the freely moving rat. Synapse, $35(2)$, pp 120-8.

[43] Thut, G., (2014) Modulating brain oscillations to drive brain function. PLoS Biol, 12(12), p.e1002032.

[44] Wach, C., Krause, V., Moliadze, V., Paulus, W., Schnitzler, A. and Pollok, B., (2013) Effects of $10 \mathrm{~Hz}$ and $20 \mathrm{~Hz}$ transcranial alternating current stimulation (tACS) on motor functions and motor cortical excitability. Behavioural brain research, pp 241, 1-6.

[45] Zaehle, T., Rach, S. and Herrmann, C.S., (2010) Transcranial alternating current stimulation enhances individual alpha activity in human EEG. PloS one, 5(11), p e13766. 